\title{
Psychological pain and previous suicide attempts in young adults: Results with the Portuguese version of the Psychache Scale
}

\author{
Rui C. Campos ${ }^{1}$ (1) | Ronald R. Holden ${ }^{2}$ (1)
}

\footnotetext{
${ }^{1}$ Departamento de Psicologia, Escola de ciências Sociais e Centro de Investigação em Educação e Psicologia (CIEP-UE), Universidade de Évora, Évora, Portugal

${ }^{2}$ Queen's University, Kingston, Canada
}

\section{Correspondence}

Rui C. Campos, Departamento de Psicologia, Universidade de Évora, Apartado 94, 7002-554 Évora, Portugal.

Email: rcampos@uevora.pt

Funding information

Fundação para a Ciência e a Tecnologia,

Grant/Award Number: UID/CED/04312/2019

\begin{abstract}
Objectives: In this study, we tested whether the unbearable pain scale of the Psychache Scale provides value for the statistical prediction of a previous suicide attempt; we evaluated whether psychological pain has incremental value in the statistical prediction of a previous suicide attempt relative to the reporting of having had a psychiatric diagnosis, and we established a cut-score for the Psychache Scale.

Method: Two samples participated: a sample consisting of 1,460 young adults and a sample of 628 Portuguese community adults.

Results and Conclusions: Results demonstrated that unbearable pain is not a stronger predictor of a previous suicide attempt than is the bearable pain score of the Psychache Scale; that the total score of the Psychache scale provides an additional statistically significant contribution to the statistical prediction of a previous suicide attempt relative to the reporting of having had a psychiatric diagnosis.
\end{abstract}

KEYWORDS

cut-score, previous suicide attempt, Psychache Scale, psychiatric diagnosis, unbearable pain 